\title{
A framework for psychological interventions in Autism Spectrum Disorder
}

\author{
Joachim Diederich \\ American University of Beirut \\ Department of Psychology \\ Riad El-Solh / Beirut 11072020 \\ Lebanon \\ jd43@aub.edu.lb
}

\begin{abstract}
Psychological interventions for autism spectrum disorder (ASD) aim at shaping behaviour by use of classical or instrumental conditioning or try to address co-morbid mental health conditions by use of cognitive behaviour therapy or other approaches. Few psychological interventions directly address deficits in ASD such as social communication issues or repetitive behaviours. The objective here is to provide a framework for psychological interventions that directly address core deficits within the spectrum and also help with mental health and behavioural issues closely associated with it. From a research perspective, the framework allows the identification of gaps where additional psychological techniques are required and facilitates strategies for empirical research. For the clinical practitioner, a structure for psychological interventions is provided in order to facilitate the search for treatment options.
\end{abstract}

\section{Introduction}

There is no coherent framework for psychological therapy available at this point in time. Heuristic search, an important method in artificial intelligence (AI), can serve as a general frame of reference for psychotherapy. This approach is based on a clear definition of the starting and end points of psychological therapy and how to achieve identified outcomes; it allows the utilisation of particularly effective psychological interventions and a ranking of different forms of psychotherapy with regard to time and effort. A cornerstone of this framework is the notion of "deep heuristic", that is, particularly effective psychological interventions. It is likely that the heuristic search framework can be applied to interventions in psychiatry as well.

Heuristic search is a form of problem solving. There is a clearly identified start state (e.g. a client with a set of symptoms) and a goal state (e.g. the client is free of symptoms). Often there are multiple or complex goals (e.g. client is free of symptoms and well supported by a social network). There are also means of transforming one state to another: psychological interventions. These interventions are informed by evidence-based research but clinical practice is important as well. A significant part of the training of clinical psychologists is based on clinical practice. An experienced clinician develops heuristic knowledge; facts and methods that are particularly useful. Sometimes this knowledge is explicit, for instance, when the therapists knows that s/he can initiate a certain action; sometimes the knowledge is implicit, that is, there is no conscious 
awareness of it. Here, deep heuristics refer to psychological interventions that are deemed particularly useful in a given context.

The framework below structures possible psychological interventions specifically for autism spectrum disorder; techniques that aims at achieving goals such as developing empathy and tolerating change in life. Table 1 and the sections below include sample interventions to illustrate the usefulness of the framework. No claim is made about the efficacy or effectiveness of individual interventions.

The goal of psychological therapy is to support individuals with social communication issues as well as behavioural problems such as an overly restricted area of interest or repetitive behaviours that interfere with normal functioning. In this sense, the framework intends to address core deficits within the broad spectrum of autism. However, within the general debate of "autism cure" versus "autism rights", the position here is to support individuals with ASD if requested.

\begin{tabular}{|c|c|c|c|}
\hline $\begin{array}{c}\text { Cognitive Functioning / } \\
\text { Therapy }\end{array}$ & Low & Medium & High (Aspergers) \\
\hline \multicolumn{4}{|l|}{ Individual } \\
\hline Pivotal response training (ABA) & Client oriented & & \\
\hline Discrete trial training (ABA) & $\begin{array}{l}\text { Shape } \\
\text { Behaviour }\end{array}$ & & \\
\hline Psychoeducation & & What is ASD? & What is Aspergers? \\
\hline Problem Solving Therapy & $\begin{array}{l}\text { Means of } \\
\text { dealing with } \\
\text { problems }\end{array}$ & $\begin{array}{l}\text { Making } \\
\text { effective } \\
\text { decisions } \\
\end{array}$ & $\begin{array}{l}\text { Goal setting and } \\
\text { identification of } \\
\text { obstacles }\end{array}$ \\
\hline Cognitive Empathy Training & & Facial Videos & Facial Videos \\
\hline \multicolumn{4}{|l|}{ Social } \\
\hline Behavioural Feedback & & Geek Cards & $\begin{array}{l}\text { Geek Cards and } \\
\text { behaviour analysis }\end{array}$ \\
\hline Stability and Change & $\begin{array}{l}\text { Manage } \\
\text { transitions }\end{array}$ & $\begin{array}{l}\text { Manage } \\
\text { transitions }\end{array}$ & Workbooks \\
\hline Positive Communication & Skill training & $\begin{array}{l}\text { Using all } \\
\text { channels }\end{array}$ & Using all channels \\
\hline Relaxation & $\begin{array}{l}\text { Progressive } \\
\text { Muscle } \\
\text { Relaxation } \\
\end{array}$ & $\begin{array}{l}\text { Progressive } \\
\text { Muscle } \\
\text { Relaxation } \\
\end{array}$ & Autogenic Training \\
\hline Psychodrama & & Modelling & Modelling \\
\hline
\end{tabular}

Table 1: A framework for psychological interventions for Autism Spectrum Disorder.

This framework for autism spectrum disorder includes three core dimensions: (1) From individual to group interventions (including partnerships) and (2) from the behavioural to the cognitive, that is from Applied Behavioral Analysis (ABA) style interventions to cognitive 
therapy informed by recent research results. The third dimension is the level of cognitive functioning, from non-verbal autistic individuals with reduced intellectual functioning to adult Aspergers with average or above average intelligence. It is possible to locate a number of psychological interventions within this framework. Given that a significant number of individuals with autism spectrum disorder also suffer from mood disorders, particularly depression and anxiety, interventions that address mood issues are considered as well.

\section{The framework}

This framework is primarily aimed at supporting adolescent and adult clients. Most importantly, the social interventions include partners and family members as well.

Some of the sample interventions in Table 1 are briefly summarised below.

\section{$\underline{\text { Psychoeducation }}$}

Education is a crucial aspect of any psychological therapy. Psychoeducation for ASD should include common challenges as well as some information on the prevalence of ASD. This may include the information below which focusses on challenges, not diagnostic definitions.

Asperger is one of the autism spectrum disorders. Individuals with Asperger syndrome often experience a number of problems: Appropriately recognising and communicating emotions, starting and maintaining friendships and relationships, finding and keeping a job, joining a network of colleagues and negotiating problems in the family or in relationships. The correct assessment and diagnosis of the condition is important, followed by psychological support and therapy.

\section{Problem-Solving Therapy}

Traditionally, problem-solving therapy includes adaptive problem-solving skills to cope with a stressful problem (American Psychological Association, Division 12, 2018). However, it is possible to go much further and see problem solving as a general framework for psychological therapy.

While psychological assessment, and in particular psychometric testing, has a firm theoretical, mathematical basis, no such coherent and unifying framework is available for psychological therapy. Heuristic search, an important method in artificial intelligence, can serve as a general framework for psychotherapy. A heuristic is a "rule of thumb", a method that might not always find the best solution but is guaranteed to find a good solution in reasonable time. Heuristic search is a form of problem solving that aims at finding the best and most cost-effective path from a starting point to a well-defined goal. This approach is based on a clear definition of the starting and end points of psychological therapy and how to achieve identified outcomes; it allows the utilisation of particularly effective psychological interventions and a ranking of different forms of psychotherapy with regard to time and effort. A cornerstone of this framework is the notion of "deep heuristic", that is, particularly effective psychological interventions.

Daily clinical practice includes the observation of the behaviour of a client, partners, care-givers 
and families. It includes the understanding and appropriate response to facial expressions, gestures, the use of metaphors and sometimes slang language. Much of this knowledge is embodied in the sense that clients use their full presence to communicate and the psychologist is using speech, voice, gesture etc. to ask questions and to conduct psychological interventions.

As a result, the "knowledge base" of a clinical psychologist is not only a large network of facts but "a large array of informal judgement rules (heuristics) which guide the system (here: the clinical psychologist) toward plausible paths to follow and away from implausible ones (Lenat, 1982, p.189). This path includes the individual steps of psychological assessment and therapy. Furthermore, this heuristic knowledge cannot be directly observed, however, it frequently is the foundation of daily, clinical practice.

For instance, an experienced psychologists may use "goal decomposition" as a strategy within the general context of problem-solving therapy. Based on clinical experience and an understanding of the problem, the psychologist may suggest sub-goals in order to achieve the overall outcome (the goal). If the psychologist has worked with clients who are on the autistic spectrum, the practitioner may may well have a set of heuristics that can help to achieve subgoals as well as the final outcome.

"Geek cards": Feedback on behaviours.

There are informal reports about the use of "Geek cards" at a computer conference several years ago. According to these reports, female participants who were in the minority felt mistreated by some of the male participants and started to hand out cards in different colours: red, yellow and green (see Geek Feminsim Wiki, 2018). The red card was supposed to mark completely inappropriate behaviour, the yellow card was used to indicate behaviour that was still inappropriate but forgivable while the green card was used to comment other participants on positive and polite behaviours that are welcomed and should be repeated.

In terms of learning theory, the "Geek cards" are markers for behaviours designed to overcome the credit assignment problem, that is how to identify a particular action within a flow of behaviours that deserves positive reinforcement or is marked as unwanted. Obviously, in the context of behavioural therapy, none of the cards should be punishing but should encourage behaviour modification.

The cards can be used as a form of behaviour feedback in autism spectrum disorder, for instance within a family or a relationship. Participants distribute cards in different colours (red, yellow and green) to mark behaviours of a partner or relative. The red card points to an unacceptable behaviour, the yellow card to actions that are not positive and should be avoided and the green card is a marker for positive reinforcement, that is it indicates behaviours that are welcomed and should be repeated.

There should be no discussions of behaviours when cards are exchanged (otherwise there could be arguments). The review of behaviours should happen later in the day and outside of the context of the occurrence of a particular behaviour. 
Naturally, there can be a technical solutions for "Geek cards". For instance, in a relationship both partners could use a mobile app that has red, yellow and green buttons. When pressed a time stamp is used to mark the behaviour and maybe the possibility for brief comment is provided as as well.

\section{Positive Social Communications: Using all the channels.}

Individuals with autism spectrum disorder may have deficits in expressing positive emotions and sentiments through body language, gestures and aspects of speech such as intonation. As a result, these individuals may be perceived as cold and disinterested. It is important to encourage persons with ASD to communicate positive emotions through all possible channels, in part to compensate for deficits that may exist in the non-verbal domain. All possible channels includes the use of social media and mobile apps to communicate positive sentiments to partners or family members. This can be done by the use of SMS, Twitter, Facebook or any other form of textual or visual communication. The idea is to stay in touch with relevant others throughout the day and to establish a constant flow of positive comments in order to maintain relationships. All of these messages can be brief, however, the idea is to communicate frequently. Initially, these messages may be scheduled at certain times throughout the day in order to establish a pattern.

\section{Stability and change: What did I expect to change?}

Insistence on routines (sameness) and intolerance of change are among the diagnostic criteria for autism spectrum disorder (Lawson, Mathys \& Rees, 2017). Individuals with ASD are less surprised than neurotypical adults when their expectations are violated.

How many things in daily life do change and how many remain the same? It is helpful to record the frequency of daily change, this includes events at home, at work, in the outside environment and in relationships. This is very much the style of cognitive behaviour therapy workbooks. Did your partner move things again in your bedroom or study? Was the traffic really as bad as predicted? Did the rearrangement of desks in the office take place?

Maybe there are not as many changes as expected and hence the knowledge of stability can and should be reassuring. Table 2 demonstrates the recording of events that break routines, the strength of the belief that these things will happen and the confirmation or absence of change for any given day.

\begin{tabular}{|l|l|l|l|}
\hline Date & Expected to change & Strength of belief (0-10) & Confirmed change \\
\hline & & & \\
\hline & & & \\
\hline & & & \\
\hline
\end{tabular}

\section{Table 2: The recording of change.}

Some of the changes in life are simply unavoidable, others can be controlled at least to some extent. The amount of traffic on the way to work cannot be controlled, however, it is possible to talk to colleagues and managers about timetables and the planning of events at work. If change 
that breaks routines goes ahead, it is useful to know well in advance and to plan for it. Table 2 can be expanded to include a classification of events: those that cannot be controlled by an individual and do not allow for any planning, those that cannot be controlled but are predictable and those events that are both foreseeable and open to individual influence.

\section{Conclusion}

This approach here is based on a clear definition of the starting and end points of psychological therapy and how to achieve identified outcomes; it allows the utilisation of particularly effective psychological interventions and a ranking of different forms of psychotherapy with regard to time and effort. The framework for ASD outlined above includes three dimensions: (1) From psychological interventions for individuals to psycho-social forms of therapy, (2) from behavioural interventions to cognitive behaviour therapy, and (3) from low to high-cognitive functioning. A number of interventions have been identified within this framework and the objective is to use the classification as a guide for future research.

\section{References}

Geek Feminism Wiki. http://geekfeminism.wikia.com/wiki/Creeper_Move cards Retrieved 22 March 2018.

Lawson, R.P., Mathys, C., Rees, G. Adults with autism overestimate the volatility of the sensory environment. Nature Neuroscience 20 (2017) 9, 1293-1299.

Lenat, D.B., The Nature of Heuristics. Artificial Intelligence, 19 (1982) 189-249.

What is Problem-Solving Therapy? American Psychological Association | Division 12 http://www.div12.org/sites/default/files/WhatIsProblemSolvingTherapy.pdf Retrieved 22 March 2018. 